\title{
Effectiveness of Emotion Regulation Training and Assertiveness Training on Resilience and Clinical Symptoms of Students with Generalized Anxiety Disorder
}

\author{
Masoud Ahmadi ${ }^{\circledR}$, Masoud Ghasemi* ${ }^{(\mathbb{D}}$, Mansoureh Shahriari Ahmadi ${ }^{\circledR}$ \\ Faculty of Educational Sciences and Psychology, Islamic Azad University, Central Tehran Branch, Iran
}

\begin{abstract}
Background: Generalized anxiety disorder is among the most common psychiatric disorders. We aimed to compare the effectiveness of emotion regulation training and assertiveness training on resilience and clinical symptoms of students with generalized anxiety disorder.

Methods: This study was a quasi-experimental pretest-posttest study with a control group. The statistical population included all 16-18 years old male high school students in Karaj who were studying in the academic year 2019-2020, of whom 75 were selected by multi-stage random sampling and assigned to two groups of 25. Participants in experimental group 1 received eight sessions of assertiveness training for 60 minutes per week. Participants in experimental group 2 received five sessions of emotion regulation training for 60 minutes per week, and participants in the control group did not receive any intervention. The Generalized Anxiety Disorder and Resilience questionnaires were used to collect data.

Results: Multivariate analysis of covariance (MANCOVA) showed that both assertiveness and emotion regulation training programs increased resilience and reduced clinical signs of generalized anxiety disorder in the experimental groups $(P<0.001)$. Moreover, there was no significant difference between the effectiveness of the two training programs.

Conclusion: It is suggested that assertiveness and emotion regulation training be provided for students and included in their curriculum, to enhance assertiveness and emotion regulation skills. Keywords: Assertiveness training; Emotion regulation training; Resilience; Clinical symptoms; Generalized anxiety disorder
\end{abstract}

*Correspondence to Masoud Ghasemi, Assistant Professor, Faculty of Educational Sciences and Psychology, Islamic Azad University, Central Tehran Branch, Iran. Email: mas. qasemi@iauctb.ac.ir

Published online 30 October 2021

Citation: Ahmadi M, Ghasemi M, Shahriari Ahmadi M. Effectiveness of emotion regulation training and assertiveness training on resilience and clinical symptoms of students with generalized anxiety disorder. Clin Neurosci J. 2021;8(4):188-192. doi:10.34172/ icnj.2021.37.

\section{Introduction}

Anxiety disorders are one of the most common disorders among adolescents. ${ }^{1}$ Generalized anxiety disorder is a chronic anxiety disorder characterized by severe and uncontrollable anxiety and associated physicalpsychological symptoms. These individuals report degrees of performance impairment ranging from job focus to interpersonal relationships. ${ }^{2}$

When a person becomes anxious, their anxiety level increases sharply, this progress reduces their psychological well-being. Resilience protects people safe from pathological disorders and life problems and helps the person cope with and adapt to difficult and stressful life events. ${ }^{3}$ Resilience is defined as retaining competence in threatening and stressful situations and returning to the original state after experiencing a psychological trauma. ${ }^{4}$

Various methods have been used to treat psychological damage in students with generalized anxiety disorder. One of these therapies that has been shown to be effective in increasing resilience and reducing anxiety in normal and clinical populations is assertiveness training. ${ }^{5}$ Expression refers to behavior that enables a person to act in favor, to express his or her true feelings honestly, and to claim rights by respecting the rights of others. ${ }^{6}$ The goal of assertiveness training is to increase the mental and practical skills of appropriate assertiveness behaviors in students. ${ }^{7}$ During the training sessions, the instructor helps students deal appropriately with various issues and various life situations, as well as to communicate more effectively with the environment. It also increases the feeling of satisfaction and the probability of receiving reinforcement from social environments and people experience a feeling of freshness and vitality during the day. ${ }^{8}$

(C) 2021 The Author(s). This is an open access article distributed under the terms of the Creative Commons Attribution License (http:// creativecommons.org/licenses/by/4.0/), which permits unrestricted use, distribution, and reproduction in any medium, provided the original work is properly cited. 
Various studies have been done regarding the use of assertiveness training as a therapeutic method. Ramadhan and colleagues confirmed the effectiveness of assertiveness training on students' resilience. ${ }^{9}$ Another study showed the effectiveness of assertiveness training in reducing social anxiety in female students. ${ }^{5}$ One other study showed the effectiveness of assertiveness training on the anxiety syndrome of futsal players. ${ }^{10}$

Other treatments that have been shown to increase resilience in normal and clinical populations such as emotion regulation training. ${ }^{11}$ Emotion regulation training means reducing and controlling negative emotions and how to use positive emotions. ${ }^{12}$ Emotion regulation increases human ability after negative stressful emotional experiences and helps the person to adapt to the environment better. ${ }^{13}$

Teaching emotion regulation to individuals may help with generalized anxiety disorder, and increase the capability to regulate emotions after facing stressful events. ${ }^{13}$ In this regard, Wang and colleagues ${ }^{14}$ showed the effectiveness of emotion regulation training on anxiety disorders. Another study showed that emotion regulation techniques reduced negative emotional responses and thus increased a person's resilience in adverse conditions. ${ }^{15}$

Most epidemiological studies in Iran have identified generalized anxiety disorder as the most common anxiety disorder. ${ }^{2}$ It is one of the most important disabling disorders for adults. ${ }^{16}$ Currently, considering the psychological damage in students with generalized anxiety disorder and the need to use appropriate and timely intervention methods and treatment, and on the other hand, by observing the effectiveness of assertiveness training and emotion regulation in improving resilience in different statistical communities, we aimed to compare the effectiveness of assertiveness and emotion regulation training programs on resilience and clinical signs of students with generalized anxiety disorder symptoms.

\section{Materials and Methods}

The present study was a quasi-experimental study with pre- and post-test with a control group design. The statistical population of the study included all male students aged 16-18 years studying in the second year of high school in Karaj in the academic year 2019-2020. Multi-stage random sampling was performed. Thus, after estimating the sample size with Cochran's formula, four schools from four districts were randomly selected, then four classes from each school and 25 students from each class were randomly selected as the prototype and the Comprehensive Anxiety Disorder Questionnaire was given to them. In the next stages, 75 students were selected as the main sample and randomly assigned to the experimental and control groups. The inclusion criteria were: high score in the general anxiety disorder questionnaire, conscious consent to participate in the study, not participating in similar psychological interventions. We excluded those who were not willing to continue and were absent for more than two sessions. Then, for the first experimental group, assertiveness training was performed in 8 sessions of 60 minutes per week and for the second experimental group, emotion regulation training was performed in five weekly 60-minute sessions, and at this time, the control group did not receive any intervention. At the end of the intervention sessions, the general anxiety disorder questionnaires and the resilience questionnaire were administered as a post-test in all three groups and the results of the three groups were compared.

The following tools were used to collect information:

\section{Generalized Anxiety Disorder Questionnaire (GAD7)}

The tool is a seven-item anxiety scale developed by Spitzer and colleagues. To identify possible cases of generalized anxiety disorder and assess the severity of its symptoms. Answer options include no, several days, more than half a day, and almost every day, which are scored from 0 to 3 , respectively. The total score of the scale is obtained by adding the scores of its questions and has a range between zero and 21. Its cut-off point is a score of 10 or higher, which provides a logical cut-off point for identifying cases of generalized anxiety disorder. The internal consistency of this questionnaire was 0.92 and the reliability of its retest was $0.83 .{ }^{17}$ In Iran, the internal consistency of this test was obtained through Cronbach's alpha of 0.85 . The correlations of this scale with Spielberger's state anxiety scale, Spielberger trait anxiety scale and clinical symptom checklist anxiety subscale were $0.71,0.52$ and 0.63 , respectively, which shows its appropriate convergent validity. ${ }^{18}$

\section{Resilience Questionnaire}

The scale was designed by Connor and Davidson in 2003 to measure people's resilience. This scale has 20 items scored on a 4-point Likert scale from completely false (zero) to always true. ${ }^{19}$ The total score in this test ranges from 0 to 100, and higher scores indicate more resilience in the person. ${ }^{19}$ Connor and Davison reported the internal stability of the resilience scale using Cronbach's alpha to be 0.89 and a retest reliability of $0.87 .{ }^{19}$ In Iran, Mohammadi calculated the reliability of the Connor and Davison scales reporting a Cronbach's alpha of 0.89 and stated that it was valid and reliable. ${ }^{20}$

In this study, the assertiveness training protocol ${ }^{6}$ was performed for the first experimental group and the emotion regulation protocol was performed based on the instructions of Allen and colleagues ${ }^{21}$ for the second experimental group. A summary of the sessions of both training programs is provided in Tables 1 and 2 .

Multivariate analysis of covariance was used to analyze the data. Data were analyzed using SPSS software, version 24 . 
Table 1. Summary of Assertiveness Training Sessions

\begin{tabular}{|c|c|}
\hline Meeting & Summary of the Content of the Meetings \\
\hline 1 & $\begin{array}{l}\text { Establishing relationships with group members, Familiarization with group work and familiarity with the concept of assertiveness and how } \\
\text { assertiveness can help improve people's quality of life. Pre-test run }\end{array}$ \\
\hline 2 & $\begin{array}{l}\text { Introducing the benefits of assertiveness to students, introducing the functions of assertiveness to students and expressing the harms of } \\
\text { assertiveness }\end{array}$ \\
\hline 3 & $\begin{array}{l}\text { Creating the ability to recognize individual rights, recognize social rights, achieve their inalienable rights without violating the rights and rights } \\
\text { of others and explain the concept of self-esteem and the need for its importance in individual and social relations. }\end{array}$ \\
\hline 4 & Familiarity of students with assertive behavior and its difference with other behaviors \\
\hline 5 & Identify the underlying thoughts of behavior and mental presuppositions, identify the underlying feelings of behavior \\
\hline 6 & Introduce students to effective ways to combat negative thoughts \\
\hline 7 & Active listening skills training to students \\
\hline 8 & Teaching the student effective techniques in relation to constructive and non-constructive criticism \\
\hline
\end{tabular}

Table 2. Summary of Emotion Regulation Intervention Sessions

\begin{tabular}{ll}
\hline Meeting & Summary of the Content of the Meetings \\
\hline 1 & Communication, conceptualization, the need to use emotion regulation training, awareness of emotions, types of positive and negative emotions. \\
2 & Training to accept emotions without judgment, cognitive errors in emotions, how to control negative emotions such as anger \\
3 & Emotional situation assessment training, proper expression of emotions. \\
4 & Emotional intelligence, emotional self-awareness, empathetic ability of interpersonal relationships. \\
5 & Emotion management through stress management, types of stress, personality measures C, B, A and methods of coping with stress. \\
\hline
\end{tabular}

\section{Results}

Mean \pm SD age of the participants in the experimental and control subjects were $2.08 \pm 16.76$ and $16.44 \pm 1.96$, respectively.

Table 3 shows the mean and standard deviation of clinical symptom and resilience scores in the experimental and control groups.

Before performing the analysis of variance with repeated measurements, the assumptions of normal distribution, homogeneity of variance, spherical hypothesis and variance-covariance matrix were calculated. Levene's test was not significant for any of the criterion variables $(P \geq 0.05)$, so the assumption of variance homogeneity of scores was assumed. Finally, Kolmogorov-Smirnov test $(P \geq 0.05)$ showed a normal distribution of data. Also, Mauchly's test of sphericity showed that the sphericity hypothesis was not observed for the group-related effects $(P<0.05$. The results of Box's $M$ test indicated that the assumption of homogeneity of variance-covariance matrix was confirmed $(P \geq 0.05)$.

Table 4 presents the results of the mixed analysis of variance test on clinical signs and resilience in the experimental and control groups.

As the results of Table 4 show, after the pre-test control, there was a difference between the experimental and control groups in the variability of resilience $(\mathrm{F}=41.25$ and $P<0.001)$ and clinical signs $(\mathrm{F}=28.34$ and $P<0.001)$. The mean scores of resilience of the experimental groups compared to the control group increased significantly and the mean scores of clinical signs of the experimental groups compared to the control group decreased significantly.

Also, the results of post hoc tests showed that there was no significant difference between the effectiveness of the training program of assertiveness and emotion regulation on the mean scores of resilience and clinical symptoms, while there was a significant difference between the two treatment groups and the control group.

\section{Discussion}

The aim of this study was to compare the effectiveness of the expression and emotion regulation training program on resilience and clinical symptoms of students with symptoms of generalized anxiety disorder. The results of data analysis showed that the emotion regulation training program was effective in improving the anxiety symptoms of students with symptoms of generalized anxiety disorder. This finding is consistent with the findings of Denny ${ }^{21}$ and Wang and colleagues. ${ }^{14}$ In fact, emotional regulation skills allow people to be aware of positive and negative emotions, not escape negative emotions when faced with stressful situations, accept and express them in a timely manner, and reduce negative emotional reactions such as catastrophe and rumination. ${ }^{21}$ In fact, emotional regulation skills allow people who are emotionally vulnerable to be in the present (present time and place) and thus have a more objective understanding of the situation instead of overreacting and anxiety. Therefore, the main focus of emotion regulation therapy is on positive and negative emotions and strengthening 
Comparing Emotion Regulation and Assertiveness Training on Resilience and Clinical Symptoms

Table 3. Descriptive Indicators Scores of Clinical Signs and Resilience in Two Stages of Measurement for the Study Groups

\begin{tabular}{|c|c|c|c|c|c|c|c|}
\hline \multirow{2}{*}{ Variable } & \multirow[b]{2}{*}{ Measurement Step } & \multicolumn{2}{|c|}{ Assertiveness Training Group } & \multicolumn{2}{|c|}{ Emotion Regulation Training Group } & \multicolumn{2}{|c|}{ Control Group } \\
\hline & & Mean & SD & Mean & SD & Mean & SD \\
\hline \multirow{2}{*}{ Resilience } & Pre-exam & 38.43 & 6.82 & 36.54 & 6.32 & 42.76 & 7.18 \\
\hline & Post-test & 56.41 & 8.03 & 53.91 & 7.73 & 41.51 & 7.55 \\
\hline \multirow{2}{*}{ Clinical signs } & Pre-exam & 13.18 & 1.89 & 12.76 & 1.67 & 12.22 & 1.62 \\
\hline & Post-test & 8.57 & 1.21 & 7.67 & 1.02 & 13.04 & 1.65 \\
\hline
\end{tabular}

Table 4. Results of Mixed Resilience Analysis Test of Variance and Clinical Signs

\begin{tabular}{lccccccc}
\hline Variable & Source of Change & Total Squares & Degrees of Freedom & Average Square & F & P Value & Effect Size \\
\hline Resilience & Group & 987.62 & 1 & 1 & 987.62 & 41.25 & 0.001 \\
Clinical signs & Group & 695.23 & 695.23 & 28.34 & 0.001 \\
\hline
\end{tabular}

them. Since the emotional processes of adolescents at this age may affect their cognitive system, by knowing and being aware of these emotional processes, the cognitive system of these people can be certificated and anxiety and depression can be prevented. ${ }^{14}$

Also, the results of the present study showed that emotion regulation training program is effective in increasing the resilience of students with symptoms of anxiety disorder. This finding is consistent with other studies. ${ }^{15,22}$ It can be said that one of the obvious effects of emotion regulation training is the ability to identify and distinguish positive and negative emotions, which makes it possible for people exposed to emotions to manage their emotions while reducing their negative emotions and vice versa, and this ability in turn leads to increased resilience. ${ }^{23}$ Emotional regulation contributes to the ability to understand, adjust and experience emotions through increasing positive emotional regulation such as positive reassessment, effectively copes with stressful situations. ${ }^{24}$ In addition, emotional regulation enables a person to use an adaptive pattern to deal with negative emotions, which leads to fewer mental health problems, which in turn leads to better adaptation and greater resilience. ${ }^{24}$

We found that the assertiveness training program was effective in improving the anxiety symptoms of students with symptoms of generalized anxiety disorder. This finding is in line with the findings of Janjani and clleagues $^{5}$ and Aliyar Najafabadi and colleeagues. ${ }^{10}$ Explaining this finding, it can be said that assertiveness training is one of the behavioral interventions that is used to improve a person's adaptation to the environment and causes satisfaction. Assertiveness training program in students with low expression, makes them more aware of their behavior and enables them to have more appropriate behavioral reactions to those around them. learning assertiveness behavior equips one with the appropriate tools for social interaction and reduces the anxiety of honestly expressing and rejecting the unreasonable demands of others. ${ }^{5}$

Also, the results of the present study showed that the assertiveness training program was effective in increasing the resilience of students with symptoms of anxiety disorder. This finding is in line with the findings of previous studies. ${ }^{9,25}$ In assertiveness training program, a kind of insight is created in the person in relation to the type of interactions they had so far, and it empowers people to have more control over situations, to take responsibility for their behaviors, and to act boldly. Since the quality of people's social interactions has a direct impact on their quality of life, enriching social relationships will be an important and effective way to increase resilience. ${ }^{26}$ Thus, assertiveness training can help people set more realistic goals by providing self-knowledge, and makes dreams more realistic, and encourages the individual to develop and grow in other ways. ${ }^{9}$

Like other studies, the present study had some limitations. For example, due to the lack of long-term access to students, it was not possible to conduct a followup study to evaluate the continuity of effectiveness. Also, in the present study, only a questionnaire was used to measure the variables and part of the results may be related to the sensitivity of the subjects to the test questions, test performance and set norms, so it is suggested that other data collection methods such as interviews be used in future research.

\section{Conclusion}

Assertiveness and emotion regulation training can be used as effective and short-term treatments to increase resilience and reduce clinical symptoms in students with generalized anxiety disorder. Therefore, it is suggested that by designing training programs for expression and regulation of emotion and its inclusion in the curriculum of students, the necessary basis be provided to strengthen related skills.

\section{Conflict of Interest Disclosures}

The authors declare that they have no conflict of interests.

\section{Ethical Statement}

All ethical principles were considered in this study. The participants were informed about the purpose of the study and 
its implementation stages. They were also assured about the confidentiality of their information, and were allowed to leave the study whenever they wished, and if desired, the findings of the study would be available to them. The participants signed the informed consent form and had the right to leave the study at any time. This research meets guidelines for ethical conduct and report of research.

\section{Acknowledgements}

We would like to thank all participants in the research and all those who have facilitated the implementation of the research.

\section{References}

1. Godini AA, Rasouli M, Mohsenzadeh F. The effectiveness of group counseling based on metacognitive therapy and dialectic behavior therapy on reducing anxiety in boy adolescent of divorce. Int Clin Neurosci J. 2019;6(3):98103. doi: 10.15171/icnj.2019.19.

2. Huang $Y$, Zhao N. Generalized anxiety disorder, depressive symptoms and sleep quality during COVID-19 outbreak in China: a web-based cross-sectional survey. Psychiatry Res. 2020;288:112954. doi: 10.1016/j.psychres.2020.112954.

3. Walker B. Resilience: what it is and is not. Ecol Soc. 2020;25(2):11. doi: 10.5751/es-11647-250211.

4. Feldman R. What is resilience: an affiliative neuroscience approach. World Psychiatry. 2020;19(2):132-50. doi: https://doi.org/10.1002/wps.20729.

5. Janjani B, Jadidi S, Vahidifard M, Tajipour A, Amokhalili $M$. The effect of assertiveness training on loneliness and social anxiety in female students with nomophobia disorder. J Psychol Stud. 2020;16(1):91-106. doi: 10.22051/ psy.2020.27706.1992. [Persian].

6. Noh GO, Kim M. Effectiveness of assertiveness training, SBAR, and combined SBAR and assertiveness training for nursing students undergoing clinical training: a quasiexperimental study. Nurse Educ Today. 2021;103:104958. doi: 10.1016/j.nedt.2021.104958.

7. Ayhan F, Teze S, Türkmen AS. The relationship between the presence of depression and attachment styles of mothers with autistic children. Am J Fam Ther. 2021:1-13. doi: 10.1080/01926187.2021.1872044.

8. Rohsig V, de Souza AB. Assertiveness training during early undergraduate years has the potential to increase students' confidence and ability to act as patient advocates. Evid Based Nurs. 2021;24(2):40. doi: 10.1136/ebnurs-2019-103235.

9. Ramadhan I, Keliat BA, Wardani IY. Assertiveness training and family psychoeducational therapies on adolescents mental resilience in the prevention of drug use in boarding schools. Enferm Clin. 2019;29 Suppl 2:326-30. doi: 10.1016/j.enfcli.2019.04.040.

10. AliyarNajafabadiR, MeshkatiZ, BadamiR. The effectiveness of assertiveness training on bullying, competitive state anxiety and performance under pressure in futsal players. Journal of Research and Health. 2020;10(5):339-48. doi: 10.32598/jrh.10.5.21.7.98. [Persian].

11. Dixon ML, Moodie CA, Goldin PR, Farb N, Heimberg RG, Gross JJ. Emotion regulation in social anxiety disorder: reappraisal and acceptance of negative self-beliefs. Biol Psychiatry Cogn Neurosci Neuroimaging. 2020;5(1):11929. doi: 10.1016/j.bpsc.2019.07.009.
12. Kale A, Gedik Z. Quality of life in riot police: links to anger, emotion regulation, depression, and anxiety. Appl Res Qual Life. 2020;15(1):107-25. doi: 10.1007/s11482-018-9667-3.

13. Bomyea J, Ball TM, Simmons AN, Campbell-Sills L, Paulus MP, Stein MB. Change in neural response during emotion regulation is associated with symptom reduction in cognitive behavioral therapy for anxiety disorders. J Affect Disord. 2020;271:207-14. doi: 10.1016/j.jad.2020.04.001.

14. Wang SM, Lee HK, Kweon YS, Lee CT, Chae JH, Kim $\mathrm{JJ}$, et al. Effect of emotion regulation training in patients with panic disorder: evidenced by heart rate variability measures. Gen Hosp Psychiatry. 2016;40:68-73. doi: 10.1016/j.genhosppsych.2016.01.003.

15. Rubin-Falcone H, Weber J, Kishon R, Ochsner K, Delaparte L, Doré B, et al. Neural predictors and effects of cognitive behavioral therapy for depression: the role of emotional reactivity and regulation. Psychol Med. 2020;50(1):146-60. doi: 10.1017/s0033291718004154.

16. Sapra A, Bhandari P, Sharma S, Chanpura T, Lopp L. Using Generalized anxiety disorder-2 (GAD-2) and GAD-7 in a primary care setting. Cureus. 2020;12(5):e8224. doi: 10.7759/cureus.8224.

17. Spitzer RL, Kroenke K, Williams JB, Löwe B. A brief measure for assessing generalized anxiety disorder: the GAD-7. Arch Intern Med. 2006;166(10):1092-7. doi: 10.1001/archinte.166.10.1092.

18. Dadsetan P, Anari A, Saleh Sedghpour B. Social anxiety disorders and drama-therapy. Dev Psychol. 2008;4(14):11523. [Persian].

19. Connor KM, Davidson JR. Development of a new resilience scale: the Connor-Davidson Resilience Scale (CD-RISC). Depress Anxiety. 2003;18(2):76-82. doi: 10.1002/da.10113.

20. Mohammadi M, Jazayeri AR, Rafie AH, Joukar B, Pourshahbaz A. Resilience factors in individuals at risk for substance abuse. J Psychol (Tabriz University). 2006;1(23):203-24. [Persian].

21. Denny BT. Getting better over time: a framework for examining the impact of emotion regulation training. Emotion. 2020;20(1):110-4. doi: 10.1037/emo0000641.

22. Cai WP, Pan Y, Zhang SM, Wei C, Dong W, Deng GH. Relationship between cognitive emotion regulation, social support, resilience and acute stress responses in Chinese soldiers: exploring multiple mediation model. Psychiatry Res. 2017;256:71-8. doi: 10.1016/j.psychres.2017.06.018.

23. Thomas C, Zolkoski S. Preventing stress among undergraduate learners: the importance of emotional intelligence, resilience, and emotion regulation. Front Educ. 2020;5:94. doi: 10.3389/feduc.2020.00094.

24. Gürcan-Yildırım D, Gençöz T. The association of selfdiscrepancy with depression and anxiety: moderator roles of emotion regulation and resilience. Curr Psychol. 2020;15:1-14. doi: 10.1007/s12144-020-00701-8.

25. Agbakwuru C, Stella U. Effect of assertiveness training on resilience among early-adolescents. Eur Sci J. 2012;8(10):69-84.

26. Yazdi-Ravandi S, Taslimi Z, Saberi H, Shams J, Osanlo S, Nori G, et al. The role of resilience and age on quality of life in patients with pain disorders. Basic Clin Neurosci. 2013;4(1):24-30. 